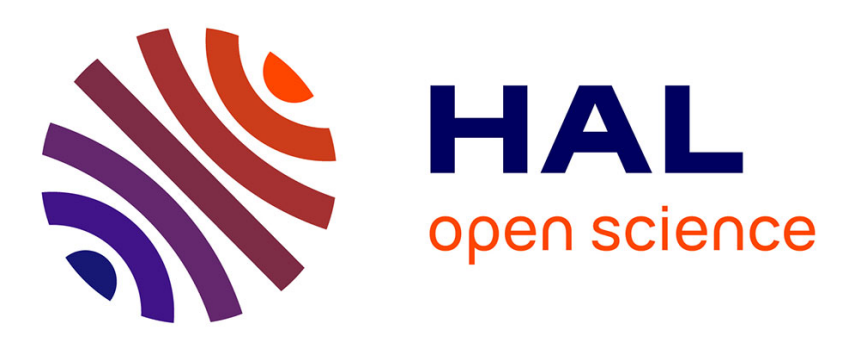

\title{
A new analytic model for the cognitive radio jump-stay algorithm
}

Michel Barbeau, Gimer Cervera, Joaquin Garcia-Alfaro, Evangelos Kranakis

\section{To cite this version:}

Michel Barbeau, Gimer Cervera, Joaquin Garcia-Alfaro, Evangelos Kranakis. A new analytic model for the cognitive radio jump-stay algorithm. WD 2013: IFIP Wireless Days, Nov 2013, Valencia, Spain. pp.1 - 3, 10.1109/WD.2013.6686443 . hal-01260581

\section{HAL Id: hal-01260581 \\ https://hal.science/hal-01260581}

Submitted on 22 Jan 2016

HAL is a multi-disciplinary open access archive for the deposit and dissemination of scientific research documents, whether they are published or not. The documents may come from teaching and research institutions in France or abroad, or from public or private research centers.
L'archive ouverte pluridisciplinaire HAL, est destinée au dépôt et à la diffusion de documents scientifiques de niveau recherche, publiés ou non, émanant des établissements d'enseignement et de recherche français ou étrangers, des laboratoires publics ou privés. 


\title{
A New Analytic Model for the Cognitive Radio Jump-stay Algorithm
}

\author{
Michel Barbeau*, Gimer Cervera ${ }^{\dagger}$, Joaquin Garcia-Alfaro $^{\ddagger}$ and Evangelos Kranakis* \\ * School of Computer Science, Carleton University, K1S 5B6, Ottawa, Ontario, Canada \\ Email: \{barbeau,kranakis\}@scs.carleton.ca \\ $\dagger$ Universidad Tecnológica Metropolitana, 97279, Merida, Yuc., Mexico \\ Email: gimer.cervera@utmetropolitana.edu.mx \\ $\ddagger$ Telecom SudParis, 91000, Evry, France \\ Email: joaquin.garcia-alfaro@acm.org
}

\begin{abstract}
In cognitive radio networks, primary users have priority over the regulated radio spectrum. Secondary users may use residual air time. We focus on the problem of meeting on a common channel by a group of secondary users. The goal is to make the users rendezvous on a common channel in a minimum amount of time. The jump-stay algorithm has been created by Lin et al. to solve this problem. We construct a new analytic model for the two-user expected time to rendezvous in the jump-stay algorithm that better reflects its performance. For the sake of comparison, we also evaluate the performance of the jump-stay algorithm through simulation.
\end{abstract}

Keywords-Channel selection, cognitive radio network, cognitive wireless network, dynamic spectrum access, jump-stay algorithm, rendezvous.

\section{INTRODUCTION}

In cognitive radio networks, secondary users can communicate over idle channels as long as they do not create interference to the primary users. In this paper, we address the problem of selecting a common communication channel, among $m$ possible channels, between a number of secondary users in a cognitive radio network, where $m$ is a positive integer. Each participant hops over a set of channels looking to rendezvous with other secondary users. Lin et al. have introduced the jump-stay rendezvous algorithm that establishes rendezvous on a common channel between an arbitrary number of secondary users [1]. Rendezvous is achieved when all users meet on a common channel, when such a channel exists. The goal is to make the users rendezvous on a common channel in a minimum number of time slots.

We revisit the work of Lin et al., the enhanced jumpstay rendezvous algorithm. For the sake of simplicity, we refer to it as the jump-stay rendezvous algorithm. While the original algorithm remains the same, we derive a new analytic model for the expected time to rendezvous (TTR) that better reflects its performance. In this short paper, the focus is on the symmetric case, i.e., a channel set common to all users. Let $p$ be the smallest prime number greater than $m$. The original analysis demonstrates that the expected TTR is lower than or equal to $\frac{3 p}{2}+3$ time slots. Our revised analysis shows that the expected TTR is lower than or equal to $p$. The new analytic model provides a significantly lower number. It is also consistent with the simulation results of Lin et al. and ours.
In Section II, we review the jump-stay rendezvous algorithm. The new analytic model of the jump-stay algorithm is developed in Section III. Simulation results are presented in Section IV. We conclude with Section V.

\section{BACKGROUND}

The jump-stay rendezvous algorithm works for multiple users with guaranteed rendezvous. We illustrate the principle with two users. Time is divided in slots of equal length. A rendezvous takes place within one time slot. It is assumed that the secondary users are synchronous. Each of them implements a cyclic behavior. It consists of four phases of the same length, in time slots. The first three phases are identical. The secondary user hops from channel to channel. All channels are visited. Each hop lasts for the duration of one time slot. During the last phase, the secondary user stays on the same channel for the whole phase duration.

Channel hopping is performed according to a pattern. Channel indices are $0, \ldots, m-1$. Let $p$ be the smallest prime number greater than $m$. For instance, if there are four channels, then $p$ is five. Hopping is performed in steps of $r$ units, with $r \in\{1, \ldots, m\}$ and starting index $i \in\{0, \ldots, p-1\}$. Each phase consists of $p$ time slots. In the first phase, hopping is performed for $p$ time slots. The same thing is done in the second and third phases. During the fourth phase, the secondary user stays on channel $r$ for $p$ time slots. The total length of a cycle, called a round from hereafter, is therefore $4 p$ time slots. Let us index the time slots with variable $t=0,1,2, \ldots, 4 p-1$. As a function of $p, r, i$ and $t$, a number pattern is generated according to the equation $j=(i+t r)$ $\bmod p$. The sequence of generated pattern numbers is such that any window of length $p$ time slots is a permutation of the numbers $0, \ldots, p-1$. The indices of the corresponding channels are produced as $c=j \bmod m$. Every channel is visited at least once during any interval of $p$ time slots.

The initial value of the step increment $r$ is selected at random. The initial value of the start index $i$ is also selected at random. It is incremented to the successor value, modulo $p$, after each round. Given a sequence generated with $r=r_{1}$ and another sequence generated with $r=r_{2}$, with $r_{1} \neq r_{2}$, then any jump pattern window of $p$ time slots of the first sequence has a common channel time slot with an overlapping jump 
pattern window of $p$ time slots of the second sequence [1]. The performance of the algorithm is evaluated in reference to the TTR metric. From the moment both users are running, it is the number of time slots required to achieve rendezvous.

\section{New AnAlytic Model}

We develop a new analytic model for the expected TTR of the jump-stay rendezvous algorithm.

Theorem 1: In the symmetric two-user case, the expected TTR of the jump-stay rendezvous algorithm is lower than or equal to $p+1 / 2$ time slots.

Proof: We assume that there are two users: User 1 and User 2. They respectively use step increments $r_{1}$ and $r_{2}$. We assume that User 2 starts when or after User 1 has started. We start counting the TTR from the time slot where User 2 has started. The analysis is structured into two main cases: when $r_{1}$ and $r_{2}$ are equal and when $r_{1}$ and $r_{2}$ are different. The probability of picking two different step increments is $\frac{m(m-1)}{m^{2}}=\frac{m-1}{m}$. The probability of picking identical step increments is $1-\frac{m-1}{m}=1 / m$.

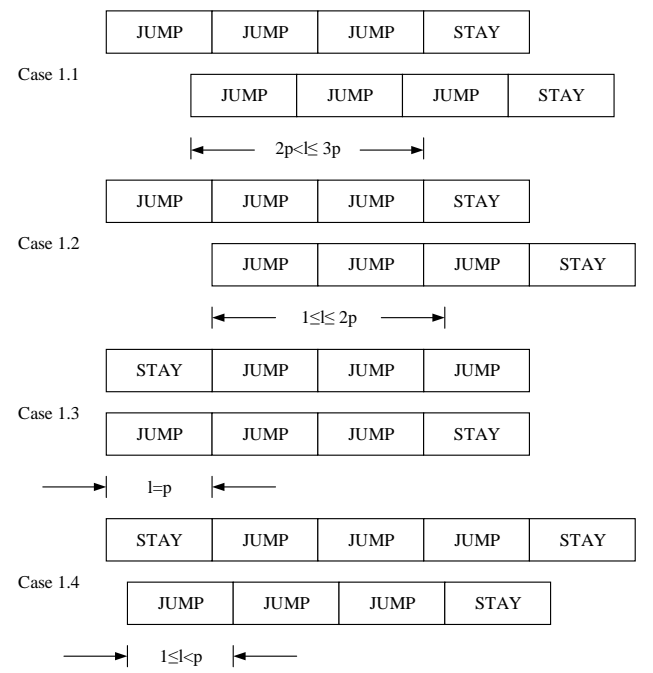

Fig. 1. Jump-stay rendezvous channel hopping Case 1, with four subcases The upper horizontal stripe represents User 1, the lower one User 2. The clear areas represent jump patterns. Grey areas are stay patterns.

Case $1\left(r_{1}=r_{2}\right)$ : There are four subcases illustrated in Figure 1.

Case 1.1: Both users are in their jump pattern and the overlap is greater than $2 p$ and lower than or equal to $3 p$ time slots. In the worst case, rendezvous takes place when they are both in their stay pattern, i.e., within a maximum of $3 p+1$ time slots from the start of User 2. Besides, there is also the non-guaranteed possibility of making rendezvous before the $3 p+1$-th time slot. Hence, each hop with index $i$ in $1, \ldots, 3 p$ can be seen as a Bernoulli trial with probability of success, i.e., rendezvous, $1 / m$ (the two users pick the same channel) and probability of failure $\frac{m-1}{m}$ (the two users pick different channels). ${ }^{1}$ For $3 p+1$-th hop, the probability of success is one, by definition of the algorithm.

\footnotetext{
${ }^{1}$ The difference between our analysis and Lin et al. is due to this assumption.
}

For a given $l$, because the sequence of Bernoulli trials is continued until the first success, the TTR, which counts the number of trials, is a random variable with

$$
\operatorname{Pr}[T T R=i]= \begin{cases}\left(\frac{m-1}{m}\right)^{i-1} \frac{1}{m} & i=1, \ldots, 3 p \\ 1-\sum_{j=1}^{3 p}\left(\frac{m-1}{m}\right)^{j-1} \frac{1}{m} & i=3 p+1 .\end{cases}
$$

The average TTR is then,

$$
\begin{aligned}
& \sum_{i=1}^{3 p} i\left(\frac{m-1}{m}\right)^{i-1} \frac{1}{m}+ \\
& \quad(3 p+1)\left(1-\sum_{i=1}^{3 p}\left(\frac{m-1}{m}\right)^{i-1} \frac{1}{m}\right)= \\
& \sum_{i=1}^{3 p} i\left(\frac{m-1}{m}\right)^{i-1} \frac{1}{m}+(3 p+1) \sum_{i=3 p+1}^{\infty}\left(\frac{m-1}{m}\right)^{i-1} \frac{1}{m} \leq \\
& \sum_{i=1}^{3 p} i\left(\frac{m-1}{m}\right)^{i-1} \frac{1}{m}+\sum_{i=3 p+1}^{\infty} i\left(\frac{m-1}{m}\right)^{i-1} \frac{1}{m}= \\
& \sum_{i=1}^{\infty} i\left(\frac{m-1}{m}\right)^{i-1} \frac{1}{m}=m .
\end{aligned}
$$

The proof uses the facts that $\left(\frac{m-1}{m}\right)^{i-1} \frac{1}{m}$ defines the probability mass function and

$$
\sum_{i=1}^{\infty} i\left(\frac{m-1}{m}\right)^{i-1} \frac{1}{m}
$$

defines the expected value of a geometric random variable with parameter $1 / m$, support $\{1,2,3, \ldots\}$ and mean $m$. This subcase occurs with probability $\frac{1}{m} \cdot \frac{p}{4 p}$, because User 2 starts between the first and $p$-th time slot from the start of User 1 .

Case 1.2: Both users are in their jump pattern and the overlap $l$ is at least one time slot, but lower than or equal to $2 p$ time slots. Consequently, there is an overlap of $p$ time slots between the stay pattern of User 1 and jump pattern of User 2 . The users make rendezvous in a maximum of $3 p$ time slots from the start of User 2. Besides, there is also the non-guaranteed possibility of making rendezvous before the $3 p$-th time slot. Hence, each hop with index $i$ in $1, \ldots, 3 p-1$ can be interpreted as a Bernoulli trial with probability of success $1 / m$ and probability of failure $\frac{m-1}{m}$. For hop $3 p$, the probability of success is one, by definition of the algorithm. Using the logic of developed for Case 1.1, the expected value is $m$ time slots. This subcase probability is $\frac{1}{m} \cdot \frac{2 p}{4 p}$, because User 2 starts from the $p+1$-th to the $3 p$-th time slot from the start of User 1 .

Case 1.3: User 1 is in the $r_{1}$ stay pattern, User 2 is in the jump pattern and the overlap is $l$ is equal to $p$. User 2 visits every channel at least once. For each time slot, we may assume that it hops on channel $r_{1}$ with equal probability. The average TTR is $\frac{1}{p} \sum_{i=1}^{p} i=\frac{p+1}{2}$. This subcase probability is $\frac{1}{m} \cdot \frac{1}{4 p}$.

Case 1.4: User 1 is in the $r_{1}$ stay pattern, User 2 is in the jump pattern and the overlap is $l$ is lower than $p$. Rendezvous takes place within a maximum of $3 p+l+1$ time slots, because they are on the same channel for sure in the $3 p+l+1$-th time slot. Each hop with index $i$ in $1, \ldots, 3 p+l$ can be interpreted as a Bernoulli trial with probability of success $1 / m$ and probability of failure $\frac{m-1}{m}$. For hop $3 p+l+1$, the probability of success is one, by definition of the algorithm. Using the logic of Case 1.1, 
the expected value is $m$ time slots. This subcase probability is $\frac{1}{m} \cdot \frac{p-1}{4 p}$. Indeed, User 2 starts when User 1 has been in the stay pattern for a least one time slot, but no more than $p$ time slots.

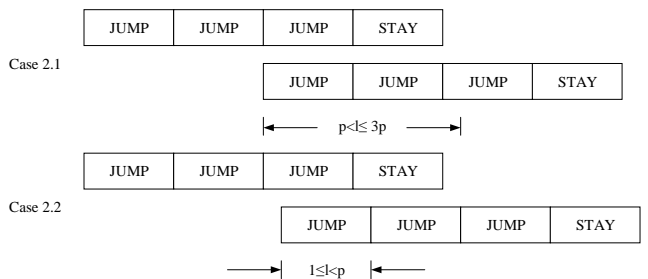

Fig. 2. Jump-stay rendezvous Case 2, with four subcases (Cases 2.3 and 2.4 are as Cases 1.3 and 1.4 in Figure 1.

Case $2\left(r_{1} \neq r_{2}\right)$ : There are four subcases illustrated in Figure 2.

Case 2.1: Both users are in the jump pattern and the overlap $l$ is greater than or equal to $p$ time slots, but lower than or equal to $3 p$ time slots. The users make rendezvous in a maximum of $p$ time slots. Assuming that they are all equally probable, on average it requires $\frac{1}{p} \sum_{i=1}^{p} i=\frac{p+1}{2}$ time slots to rendezvous. This subcase occurs with probability $\frac{m-1}{m} \cdot \frac{2 p+1}{4 p}$, because User 2 starts from the first to the $2 p+1$-th time slot from the start of User 1.

Case 2.2: Both users are in the jump pattern and the overlap is $l$ is greater than equal to one, but less than $p$ time slots. There is an overlap of $p$ time slots between the stay pattern of User 1 and jump pattern of User 2. The users make rendezvous in a maximum of $2 p-1$ time slots from the start of User 2. Assuming that they are all equally probable, on average it requires $\frac{1}{2 p-1} \sum_{i=1}^{2 p-1} i=p$ time slots to rendezvous. This subcase probability is $\frac{m-1}{m} \cdot \frac{p-1}{4 p}$, because User 2 starts from the $p+1$-th to the $3 p$-th time slot from the start of User 1 .

Case 2.3: User 1 is in the stay pattern, User 2 is in the jump pattern and the overlap is $l$ is equal to $p$ time slots. As in Case 1.3 and for the same reason, the average TTR is $\frac{p+1}{2}$. This subcase probability is $\frac{m-1}{m} \cdot \frac{1}{4 p}$.

Case 2.4: User 1 is in the stay pattern, User 2 is in the jump pattern and the overlap is $l$ is greater than or equal to one, but less than $p$. Rendezvous takes place within $2 p-1$ time slots. Assuming that they are all equally probable as in Case 2.2, on average it requires $p$ time slots to rendezvous. Start of User 2 when User 1 has been in the stay pattern for a least one time slot occurs with probability $\frac{p-1}{4 p}$. This subcase probability is $\frac{m-1}{m} \cdot \frac{p-1}{4 p}$.

Finally, the expected TTR is equal to

$$
\begin{aligned}
\frac{1}{m} \cdot\left(\frac{p+2 p+p-1}{4 p}\right) \cdot[m]+ & 1.1,1.2 \& 1.4 \\
\frac{1}{m} \cdot \frac{1}{4 p} \cdot\left[\frac{p+1}{2}\right]+ & 1.3 \\
\frac{m-1}{m} \cdot\left(\frac{2 p+1+1}{4 p}\right) \cdot\left[\frac{p+1}{2}\right]+ & 2.1 \& 2.3 \\
\frac{m-1}{m} \cdot 2 \cdot \frac{p-1}{4 p} \cdot[p]+ & 2.2 \& 2.4
\end{aligned}
$$

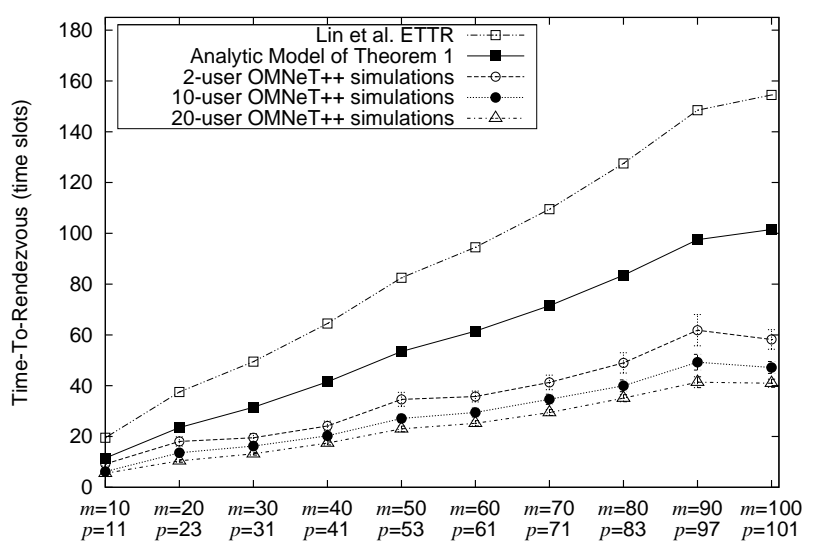

Fig. 3. Expected TTR for two-user rendezvous in 10 to 100-channel scenarios according to Lin et al., Theorem 1 and simulations.

Moreover, every term in square brackets is lower than or equal to $p$. Hence, the expected TTR is lower than or equal to $p$.

\section{Simulations}

Simulations were conducted using OMNeT++ [2], with very narrow $95 \%$ confidence intervals. Figure 3 pictures the expected TTRs in accordance to the model of Lin et al. for two-user rendezvous in 10 to 100 -channel scenarios. The TTRs are compared with the ones obtained with the analytic model presented in Section III (cf. Theorem 1). The figure is complemented with experimental results obtained with the OMNeT++ simulation. The plots confirm that the upper bound of Lin et al. is highly over estimated.

\section{Conclusion}

We have constructed a new analytic model for the expected TTR of the jump-stay algorithm of Lin et al. Their original algorithm is unchanged. The analysis is more consistent with the simulation results, the ones of Lin et al. and ours.

Lin et al. address the asymmetric case. Users have different sets of available channels. Rendezvous is possible if they are not disjoint. We also derived a new analytic model for the asymmetric case, published in a companion paper [3].

\section{ACKNOWLEDGMENT}

We acknowledge financial support from Natural Sciences and Engineering Research Council of Canada, Spanish Ministry of Science and Innovation (projects TIN2011-27076-C03-02 CO-PRIVACY and TIN2010-15764 NKHRONOUS) and Ministry of Education of Mexico. The authors thank A. Dotor for all his help on the simulations reported in Section IV.

\section{REFERENCES}

[1] Z. Lin, H. Liu, X. Chu, and Y. Leung, "Enhanced jump-stay rendezvous algorithm for cognitive radio networks," IEEE Communications Letters, pp. 1-4, 2013.

[2] A. Varga and R. Hornig, "An overview of the OMNeT++ simulation environment," in 1st International conference on Simulation tools and techniques for communications, networks and systems \& workshops (Simutools), 2008.

[3] M. Barbeau, G. Cervera, J. Garcia-Alfaror, and E. Kranakis, "A new analysis of the cognitive radio jump-stay algorithm under the asymmetric model," submitted for publication, 2014. 